Design: Cross-sectional survey of 2995 15-year-olds via questionnaire and interview, timed to collect two saliva samples for morning cortisol.

Setting: The west of Scotland (Glasgow area).

Measures: Family SES (represented by parental social class, material deprivation and family affluence); school hierarchies (derived from subjective placement on 7 "ladders" and factored into three dimensions, termed scholastic, peer and sports status); cortisol (logged to correct for skewness); biological confounds (time of awakening, time of cortisol measurement, day of the week).

Analysis: OLS linear regression (univariate and multivariate) within a multi-level (school) context, all models adjusted for biological confounds.

Results: Little or no variation in cortisol was observed for any SES measure. By contrast, each school hierarchy was independently associated with cortisol in different ways. For the scholastic hierarchy, an inverse linear relationship was found in both genders $(p<0.01)$, cortisol increasing with lower position. For peer hierarchy, an opposite linear relationship was observed for males $(p<0.001)$, cortisol increasing with higher position, while for females elevated cortisol was associated only with "top" position. For sports, elevated cortisol was only associated with "bottom" position among males, with all bar the "top" among females. Further adjustment for smoking did not alter these results.

Conclusions: These findings are interpretable against predictions about the stress correlates of hierarchical position in more and less stable social systems, the former represented by the scholastic hierarchy involving negative effects on those in lower positions, the latter by peer hierarchy having negative effects higher up. The particularly stressful "top" position for females is consistent with evidence from other studies. Overall, the results highlight the much greater importance of school-based peer groups for young peoples' stress than family SES, the latter adding to the evidence-base that youth is characterised by much less SES variation in health than any other stage in the life-course.

\section{INEQUALITIES IN THE HEALTH AND WELL-BEING OF 15-YEAR- OLD BOYS AND GIRLS IN SCOTLAND AND THE MEDIATING EFFECT OF THE FAMILY MEAL}

K Levin, C Currie. Child and Adolescent Health Research Unit, University of Edinburgh, Edinburgh, UK

doi:10.1136/jech.2009.096719|

Background: Previous research has shown the importance of the family in young people's health. In particular, family structure has been associated with a range of health outcomes with those living in both-parent families generally faring best.

Objective: The aim of this study is to describe health and health behaviour in different family structures and to assess the mediating role of the family meal on this association.

Methods: Data from the 2006 Health Behaviour in School-Aged Children survey were modelled using Multilevel Binomial modelling for boys and girls, with 14 self-report health and health behaviour outcomes, adjusting for age, family structure and family meals.

Results: Family structure was associated with boys' smoking, drinking, cannabis use, tooth brushing, life satisfaction, selfreported health, sexual intercourse and fruit consumption. Family structure was associated with girls' smoking, drinking, cannabis use, fighting, life satisfaction, happiness, self-reported health, multiple health complains (MHC), sexual intercourse and fruit and vegetable consumption. Physical activity and bullying did not differ by family structure. For the majority of outcomes, boys living in step families and girls from single parent families fared the worst. Around two thirds of young people ate a meal with their family at least 4 days per week and this was associated with positive health and health behaviour for the majority of outcomes. For example, among girls who ate 4 or more times a week with their families, the odds of smoking cannabis were 0.20 (95\% CI 0.12 to 0.33 ) those who ate with their family less often, while the odds of happiness were 1.72 (1.34 to 2.22). Similarly, among boys the odds of smoking tobacco were 0.59 (0.43 to 0.82 ) and tooth brushing 1.55 (1.21 to 2.00). Among girls, after adjusting for family meals, the association between family structure and vegetable consumption became insignificant, while the association with drinking, fighting, cannabis use, happiness and MHC was attenuated. Among boys, family meals mediated the relationship between family structure and smoking, drinking and cannabis use, and contributed to the association with sexual intercourse and fruit consumption.

Conclusions: Differences in family composition in contemporary society appear to have important implications for health outcomes of adolescents, and living with both parents is generally associated with the most favourable outcomes. While family transitions may be unavoidable, having a family meal 4 or more times a week mediates, in some cases entirely, the potential negative effects of living in single parent and step families.

\section{COMPARISON OF TEENAGERS' EARLY SAME SEX AND HETEROSEXUAL BEHAVIOUR IN THE UK: DATA FROM THE SHARE AND RIPPLE STUDIES}

${ }^{1} \mathrm{~A}$ Parkes, ${ }^{2} \mathrm{~V}$ Strange, $1 \mathrm{D}$ Wight, ${ }^{1} \mathrm{M}$ Henderson, ${ }^{1} \mathrm{~K}$ Buston. 'Medical Research Council Social and Public Health Sciences Unit, University of Glasgow, Glasgow, UK; ${ }^{2}$ Institute of Education, University of London, London, UK

\section{doi:10.1136/jech.2009.096719m}

Objectives: To compare the sexual experiences of UK teenagers who have same-sex partners with those who have exclusively opposite-sex partners, in terms of context, quality and risk involved. Pupils' background and attitudes were explored to suggest reasons for differences.

Studies: Self-complete, anonymised questionnaires were administered at ages 13/14 and 15/16 years to pupils in two school-based RCTs of sex education programmes in Scotland (SHARE) England (RIPPLE), giving a combined eligible sample of 10250 for this study. Main Measures: "First sex" (FS) reported at age 15/16 was defined for a same-sex partner as any genital contact, and for an opposite-sex partner as vaginal intercourse. Teenagers were classified according to gender of sexual partner(s) as reporting exclusively heterosexual, exclusively homosexual, or bisexual behaviour. Additional information was collected on circumstances of, and feelings after, first heteroand homosexual sex; and on heterosexual risk behaviours (age at FS, condom use, number of partners and pregnancy).

Methods: Regression models of quality of FS and of sexual risk on sexual group adjusted for age, arm of trial and gender, and took account of clustering by school.

Results: Four in ten teenagers ( $39 \%, \mathrm{~N}=3565)$ reported heterosexual intercourse and no same-sex genital contact. Same-sex FS was reported by $2.5 \%(\mathrm{~N}=201)$ teenagers. Bisexual $(\mathrm{BS})$ behaviour was reported by most $(72 \%)$ of teenagers in the homosexual behaviour group. Girls were more likely than boys to report heterosexual intercourse ( $43 \%$ vs. $35 \%$ ), but were no different in reporting samesex genital contact. First same-sex genital contact was more likely than first heterosexual intercourse to be at a younger age, unplanned, involve alcohol or drugs and no prior romantic relationship with partner. These contextual factors helped to account for lower autonomy reported for first same-sex genital contact, compared to first heterosexual intercourse. Boys and teenagers with exclusively same-sex partners were more likely than girls and teenagers with opposite-sex partners to report worse feelings after sex. Heterosexual risk was greater among teenagers who had partners of both sexes than among teenagers with exclusively opposite-sex partners. To some extent, this difference was mediated by attitudinal factors including poorer condom attitudes, as well the context of sexual behaviour. 
Discussion: Lower autonomy and greater risk-taking found among teenagers with same-sex partners echo the findings of research elsewhere (mainly the US). Further research on underlying psychosocial factors is required.

\section{CIGARETTE AND ALCOHOL CONSUMPTION AMONG YOUNG PEOPLE IN ENGLAND: FINDINGS FROM THE SMOKING, DRINKING AND DRUG USE SURVEY 2008}

D Jotangia, E Fuller. Health Research Group, National Centre for Social Research, London, UK

\section{doi:10.1136/jech.2009.096719n}

Background: The Smoking, Drinking and Drug Use Survey (SDD) among secondary school children in England provides national estimates for the proportions of young people (aged 11-15) who smoke, drink alcohol or take illegal drugs. The survey findings are used to monitor progress towards government targets for reducing smoking and alcohol misuse among teenagers. Until October 2007, it was illegal to sell tobacco products to children under the age of 16 in England. Evidence from SDD 2006 showed that despite this, children under the age of 16 could easily obtain cigarettes from shops and vending machines. From October 2007, it became illegal to sell cigarettes to young people under the age of 18. SDD 2008 is the first survey post implementation of this legislation which can provide empirical evidence against which to assess the impact of this legislative change upon young people's ability to buy cigarettes; impact upon smoking prevalence and assess whether young people have changed how and where they obtain cigarettes. The Chief Medical Officer launched a public consultation on young people's drinking, which advised that young people under the age of 16 should not drink alcohol because of the health risks associated with it. Evidence from previous SDD reports has shown a downward trend in the proportion of young people who have ever drunk alcohol; 61\% in 2003, 54\% in 2007. Results from the 2008 survey will monitor these trends and provide key estimates on young people's alcohol consumption and behaviour.

Methodology: A representative sample of pupils in school years 7 to 11, were drawn from all state-funded and independent secondarylevel schools across England. A random probability sample of both schools and pupils within schools was selected for participation in SDD 2008 and 7798 pupils in 253 schools participated. Pupils completed a self-completion questionnaire which included detailed questions about smoking and drinking consumption, and general questions about relevant behaviours, attitudes and knowledge.

Results: This is first report in the survey series to present nationally representative findings assessing the impact of regulating the sale of tobacco upon young people's smoking behaviour. Trend data about alcohol intake and frequency of consumption will also be presented. The SDD 2008 findings are currently under embargo prior to publication in July 2009.

Conclusions: The survey is a key data resource in the planning of young people's health services, education programmes and policy initiatives in England.

\section{Methods}

\section{A NOVEL APPROACH TO TESTING THE LIFECOURSE EFFECT OF BODY SIZES ON BLOOD PRESSURE IN LATER LIFE USING PARTIAL LEAST SQUARES REGRESSION}

\footnotetext{
${ }^{1,2}$ Y-K Tu, ${ }^{1} \mathrm{~A}$ Woolston, ${ }^{1} \mathrm{P}$ Baxter, ${ }^{1} \mathrm{MS}$ Gilthorpe. ${ }^{1}$ Biostatistics Unit, Centre for Epidemiology and Biostatistics, University of Leeds, Leeds, UK; ${ }^{2}$ Leeds Dental Institute, University of Leeds, Leeds, UK
}

doi:10.1136/jech.2009.096719o

Introduction: Recent studies claimed that postnatal catch-up growth might have a stronger impact on health in later life than birth size. As growth is a continuing process in lifecourse, the challenge is therefore to tease out the impact of body size in difference critical phases of lifecourse. One problem is that it is impossible to use ordinary least squares regression to differentiate the effects of birth sizes, growth and current body sizes, because growth is generally defined as the difference between birth and current size. The aim of this presentation is to propose a novel approach to testing lifecourse effects of body sizes on health outcomes using partial least squares (PLS).

Materials and Methods: Longitudinal data from a cohort of 960 males and 834 females recruited in Philippines during 1983-4 were used for statistical analysis. Body weight $z$-scores were used as the measure for body sizes, and the outcomes were systolic (SBP) and diastolic (DBP) blood pressure measured in 2002. PLS regression was used to test the impact of birth weight $z$-scores, change in body weight z-scores during different stages of growth, and current body weight $z$-scores on SBP and DBP.

Results: For SBP, birth size had a small negative association and the change in z-scores between age 1 and 2 had a stronger positive association than the change between birth and age 1. Growth after age 8 had a much stronger association than early growth, but current body size had the largest association. For DBP, birth size had a small positive association, and early growth had small associations in females. Growth after age 8 had a much stronger association than early growth, but current body size still had the largest positive association. In contrast, early growth had stronger associations with DBP in males, whilst current body size still had the largest association. Conclusion: PLS regression estimates the associations between birth sizes, changes in body sizes at different phases of lifecourse and current body sizes simultaneously, and therefore misinterpretation such as reversal paradox can be avoided. Results from PLS analysis suggested that current body size had the largest positive association with SBP and DBP, whilst birth size had small negative or positive associations with blood pressure. Later growth in childhood and adolescence had stronger associations with SBP than early growth before age 2 , but the associations between growth in body sizes and DBP were more complex.

\section{THE PREFERENCE EFFECT IN AN UNBLINDED HEALTH PROMOTION INTERVENTION TRIAL: HOW IMPORTANT IS IT?}

${ }^{1} \mathrm{C}$ Hayes, ${ }^{2} \mathrm{C}$ Collins, ${ }^{3} \mathrm{M}$ Geary, ${ }^{4} \mathrm{CC}$ Kelleher. ${ }^{1}$ Department of Public Health, Dr Steevens' Hospital, Dublin 8, Ireland; '2rish College of General Practitioners, Dublin, Ireland; ${ }^{3}$ Rotunda Hospital, Dublin, Ireland; ${ }^{4}$ School of Public Health and Population Science, University College Dublin, Dublin, Ireland

\section{doi:10.1136/jech.2009.096719p}

Background: Maternal smoking is one of the few potentially preventable factors associated with miscarriage, complications of pregnancy, low birth weight, perinatal death and poorer long term growth, development and health of the child. Health promotion initiatives pose a particular challenge especially for disadvantaged women. The preference effect, whereby people support an intervention because it conforms with their own understanding of a behaviour or disease under study, is one such determinant and is especially relevant to unblinded trials.

Objective: To determine whether assignment to intervention or control group and smoking status affected participation in an unblinded intervention study, one of the aims of which was to identify the key determinants of sustained effective brief intervention for smoking cessation in pregnancy and post partum.

Participants: The sample comprised a cohort of 1000 disadvantaged pregnant smokers who were followed up at five time pointstwice during pregnancy at the hospital, once directly after birth and at two subsequent public health nurse visits (3-4 and 7-9 months post partum). 